In Situ

Revue des patrimoines

\section{In Situ}

Revue des patrimoines

5 | 2004

Le patrimoine rural ( 1 ère partie)

\title{
Une histoire évolutive de l'habitat martiniquais
}

\section{Christophe Denise}

\section{OpenEdition}

Journals

\section{Édition électronique}

URL : http://journals.openedition.org/insitu/2381

DOI : $10.4000 /$ insitu.2381

ISSN : 1630-7305

\section{Éditeur}

Ministère de la culture

\section{Référence électronique}

Christophe Denise, « Une histoire évolutive de l'habitat martiniquais », In Situ [En ligne], 5 | 2004, mis en ligne le 19 avril 2012, consulté le 19 avril 2019. URL : http://journals.openedition.org/insitu/2381 ; DOI : 10.4000/insitu.2381

Ce document a été généré automatiquement le 19 avril 2019

\section{(c) (i) $\odot$}

In Situ Revues des patrimoines est mis à disposition selon les termes de la licence Creative Commons Attribution - Pas d'Utilisation Commerciale - Pas de Modification 4.0 International. 


\title{
Une histoire évolutive de l'habitat martiniquais
}

\author{
Christophe Denise
}

«Il faut pleurer toute case qui disparaît. Car ce n'est pas un abri qui s'en va. C'est un souvenir. C'est une histoire. C'est une manière de prendre la vie et d'organiser ce que l'on sait du monde ». Patrick Chamoiseau, Cases en Pays-Mêlés.

1 L'habitat martiniquais est le produit d'un ensemble de facteurs économiques, sociologiques, naturels très particuliers. La société coloniale et l'économie de plantation ont engendré les modèles de notre habitat traditionnel. En effet, après l'abolition de l'esclavage en 1848, la libre expression des populations a favorisé l'échange culturel, d'où l'apparition d'un style créole, d'une architecture vernaculaire, née de la fusion des cultures diverses, et formant un langage local. Malgré l'évolution observée depuis la seconde moitié du XIX ${ }^{\mathrm{e}}$ siècle, les caractères essentiels de cet habitat ont été préservés. C'est par le truchement de l'histoire des hommes et des structures (politiques, économiques) que se lit l'habitat traditionnel martiniquais. Au cœur de ce dernier se trouve la case, élément essentiel de l'habitat rural. Qu'est-ce qu'une case ? Comment a évolué l'habitat rural martiniquais? «Demeure des pauvres, et naturellement celle des petits cultivateurs $»^{1}$, son évolution est indissociable de celle de l'occupation humaine et plus généralement des mutations vécues par la société martiniquaise.

\section{Les premiers temps de la colonisation}

\section{L'habitat caraïbe}

2 A l'arrivée des premiers colons, en 1635, les cases existent déjà dans l'habitat caraïbe. Les descriptions générales montrent qu'elles sont organisées autour d'un bâtiment à usage commun de plus grande importance dans lequel se tiennent les assemblées : le carbet. D’une longueur de trente, quatre-vingts ou cent pieds, il est entouré de plusieurs autres 
cases de plus petite dimension appelées «mouinas ». Le Père Breton en fait la description suivante: «Les cases sont faites de fourches d'arbres, plantées en terre, jointes avec d'autres pièces de bois qui tiennent l'une à l'autre. Là-dessus, ils mettent des chevrons qui vont jusqu'à terre et couvrent le tout de feuilles de latanier ou de roseaux». En fait, il s'agit d'un toit à deux versants touchant le sol. Certains chroniqueurs ont aussi signalé l'existence de «mouinas » ovales. Les nouveaux habitants de la colonie se logent au départ comme les autochtones auprès desquels ils apprennent progressivement à connaître les ressources locales et les possibilités qu'elles offrent. En s'inspirant à la fois des techniques caraïbes et des ressources offertes par le territoire, cette nouvelle population élabore un habitat adapté à ses besoins.

\section{Les premières cases des colons}

3 Dans les premières années de la colonisation (1635-1650), chacun s'isole et défriche sa parcelle de terre, les bourgs et villages n'apparaissant que dans le dernier quart du XVII siècle sur le "retranchement aux 50 pas de seigneurie » réservé à cet effet par la compagnie. Par conséquent l'habitat adopte d'autres formes et la case du colon diffère dans sa conception du « mouinas », principalement utilisé pour dormir la nuit. Témoin de cette époque, le Père Jean-Baptiste Du Tertre rapporte que: «Les cases des simples habitants ne sont encore palissadées que de roseaux, particulièrement aux endroits où on ne craint pas les incursions des "sauvages ». Ces logements n'ont que des salles basses, divisées en deux ou trois départements, dont l'un sert de salle, l'autre de salle à manger, et le troisième de garde-manger. Celles des plus pauvres sont couvertes de feuilles de canne de roseau, de latanier et de palmiste. La cuisine est toujours séparée de la case. Elle est composée d'un petit appentis qui a cinq ou six pas au-dessus du vent». Comparant ces cases aux logements caraïbes, Jean-Baptiste Delawarde précise que: «La case, avec sa toiture appuyée sur des cloisons verticales est d'une silhouette toute différente. A remarquer que le foyer caraïbe est disposé dans la hutte elle-même, alors que celui des cases créoles est à l'extérieur, enfin ces dernières s'éclairent par des baies, tandis que seule une porte de deux ou trois coudées de hauteur apportait au logis indigène une faible lumière que complétait la flamme du foyer. La seule chose qui semble devoir être retenue de la tradition caraïbe est la technique du couvert, la façon d'utiliser les matériaux du pays, feuilles de latanier, de balisier, de palmiste et de roseaux qu'on fixait avec des lianes souples sur des gaulettes, pour obtenir de grandes tuiles végétales couvrant la toiture et fermant les deux extrémités de la hutte $»^{2}$.

4 L'importance de la vie à l'extérieur (cuisine séparée, dépendances), caractéristique essentielle de l'habitat traditionnel rural est sans aucun doute le principal héritage caraïbe. Durant ces vingt premières années de colonisation (1635-1655), malgré des distinctions sociales entre les habitants relativement marquées, il n'y a pas de différences fondamentales dans la manière de construire ni dans le mode d'habiter. En revanche, dans le dernier quart du XVII siècle, l'émergence de l'économie de plantation et l'institution d'un système esclavagiste vont profondément transformer le paysage martiniquais. 


\section{L'habitation-sucrerie : deux modes distincts d'habitat}

5 L'unité de base de l'économie de plantation est l'habitation-sucrerie ${ }^{3}$. Pour Danielle Bégot : «L'habitation antillaise est un domaine terrien dont la raison d'être est la mise en valeur à des fins spéculatives de terres qui ne seront jamais des terroirs, et qui a trouvé dans la culture de la canne sa plus parfaite expression $»^{4}$. Génératrice de société, elle est à la fois unité de production avec ses bâtiments, son "personnel ", et unité de base de la société esclavagiste. Par conséquent, s'il s'agit pour Danielle Bégot d'une "réalité spatiale » nécessitant une occupation et une maîtrise de l'espace des plus efficaces, c'est surtout un système organisateur, structurant la société antillaise. Dès lors, une nette distinction s'opère désormais dans l'habitat entre la maison de maître et les cases d'esclaves.

\section{Les « cases-nègres »}

Figure 1

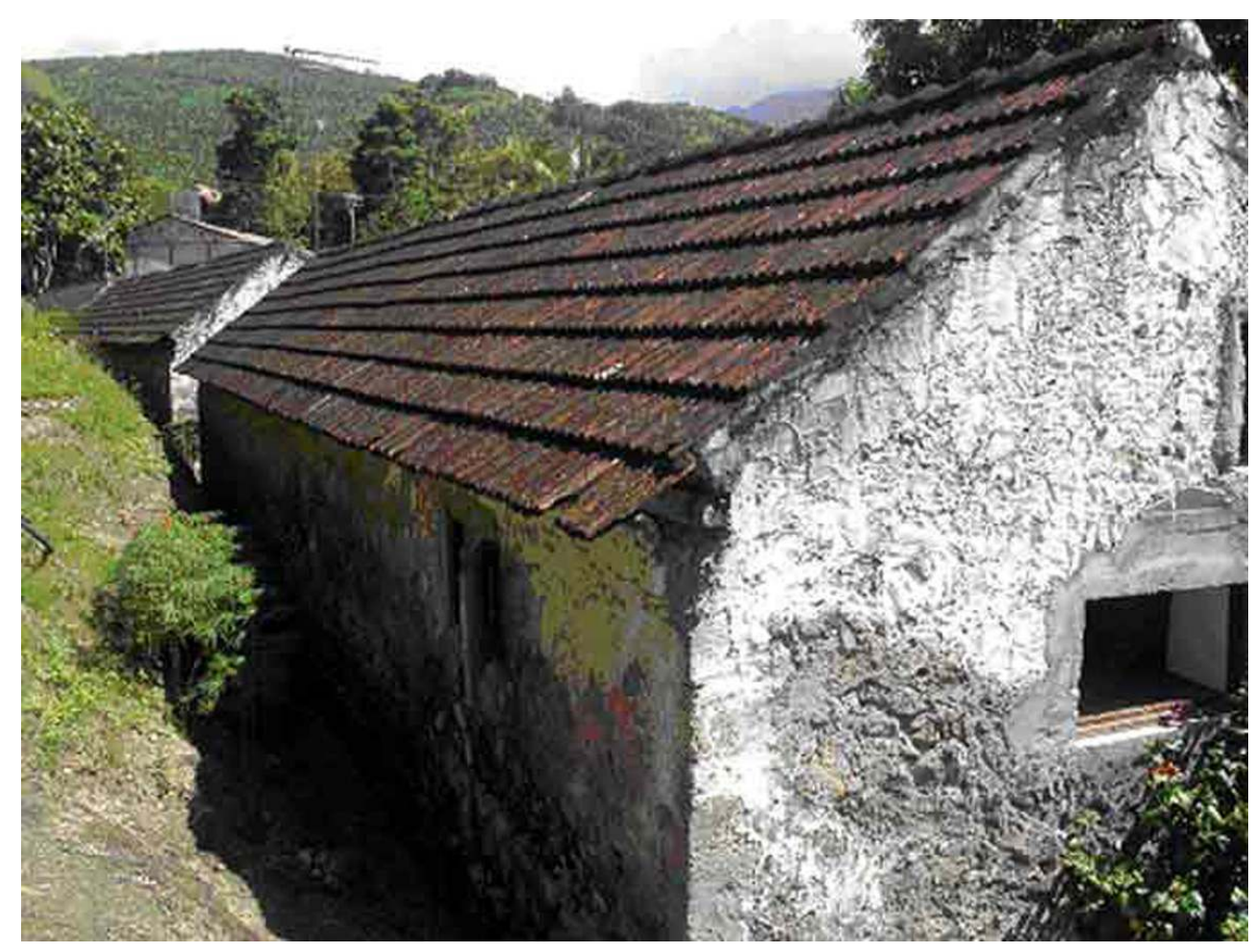

Carbet, cité ouvrière dite rue cases-nègres, habitation Lajus, fin XVIII -début du XIXe siècle Phot. Inv. O. Régis ( I) Inventaire général, ADAGP, 2003

6 Cet habitat s'inscrit dans un ensemble : « l'habitation ». Sa structure est très simple et les matériaux rudimentaires. Aussi les cases d'esclaves ne diffèrent que très peu de l'habitat des premiers colons. A ce propos, le Père Labat écrit : « (...) On leur donne pour l'ordinaire trente pieds de long sur quinze de large. Si la famille n'est pas assez nombreuse pour occuper tout le logement, on le partage en deux dans le milieu de sa longueur. Les portes qui sont aux pignons répondent sur deux rues lorsque la maison sert à deux familles (...) On couvre ces maisons avec des têtes de cannes de roseaux ou de claies faites de petites 
gaulettes pour soutenir un torchis de terre grasse ou de bouse de vache sur lequel on passe un lit de chaux (...) Elles sont regroupées (fig. $\mathbf{n}^{\circ} \mathbf{1}$ ) en un quartier unique et sont disposées géométriquement en files séparées par des ruelles». Quelques changements (solin en maçonnerie, passage à la chaux des murs montés en torchis) vont apparaître au cours $\mathrm{du} \mathrm{XVIII}^{\mathrm{e}}$ siècle sur certaines habitations. L'ensemble reste toutefois particulièrement sommaire. Evoquant un dessin à la plume de 1720 conservé au Centre des archives d'Outre-mer à Aix-en-Provence représentant l'habitation Anse Latouche, Jacques Petitjean-Roget indique que: «Vingt-quatre cases de travailleurs sont réparties en désordre de l'autre côté de la rivière, à flanc de morne. Elles sont toutes semblables avec une seule porte $»^{5}$. Avec l'habitation s'opère un changement significatif du statut de la case qui devient le logement de l'esclave et par extension du pauvre, construit à l'économie dans un souci de rentabilité maximale.

\section{La maison de maître}

7 La maison de maître est à plus d'un titre l'élément dominant de l'habitation. D'abord par sa localisation en hauteur permettant de surveiller la plupart des dépendances puis par son rôle symbolique au sein de l'habitation. « Si les bâtiments industriels constituent la raison d'être de l'habitation-sucrerie, la maison de maître en est l'agrément et, symboliquement, l'élément le plus représentatif ${ }^{6}$. Jusqu'au XIX ${ }^{e}$ siècle, elle va évoluer aussi bien sur le plan des matériaux que du parti architectural. En effet, le Père Du Tertre signale que: «Les maisons des officiers et des riches planteurs ne sont encore pour la plupart que de charpenteries revêtues de planches, avec un étage au-dessus de la salle dont le plancher est de brique. Elles sont couvertes de tuiles que les Hollandais apportent avec ces briques (...) les autres ne sont recouvertes que d'essentes de bois faites en façon de tuile $»^{7}$. Avec l'essor du sucre, les planteurs construisent leurs maisons en maçonnerie. «Les pierres assemblées à l'aide de mortier (composé d'un mélange de cendres, sirop de canne, sable, chaux) donnaient des murs épais, l'objectif étant manifestement de construire pour durer. Elles comportaient en général un corps central maçonné de forme rectangulaire, s'élevant sur deux niveaux avec un étage en bois, comprenant une grande salle au rez-de-chaussée, ceinturée de deux galeries dont l'une, couverte, pouvait abriter la cuisine et la réserve; un escalier menait aux combles qui contenaient les chambres, la toiture, souvent en pente était recouverte de tuiles $»^{8}$. C'est au cours du XIX ${ }^{e}$ siècle que furent construites les maisons de maitre en bois sur un soubassement de maçonnerie, avec galerie. On peut alors parler de «style colonial ».

8 Le dernier quart du XIX siècle et la première moitié du $\mathrm{XX}^{\mathrm{e}}$ siècle n'apportent pas de changements importants dans la conception de ces deux modèles qui caractérisent l'habitat rural traditionnel.

\section{Description et caractères essentiels de la case traditionnelle martiniquaise}

Avec le morcellement de la propriété foncière et l'occupation des terres (seconde moitié du XIX ${ }^{e}$ siècle), elle devient un bien propre, chaque habitant construisant sa case sur sa parcelle de terre. Après l'abolition de l'esclavage, «le peuple qui hérite d'un savoir-faire et d'usages a désormais l'initiative et la responsabilité de son habitat ${ }^{9}$. Pour Jack Berthelot, l'histoire de la case ne débute réellement qu'à cette période. 


\section{La case et ses dépendances}

Figure 2

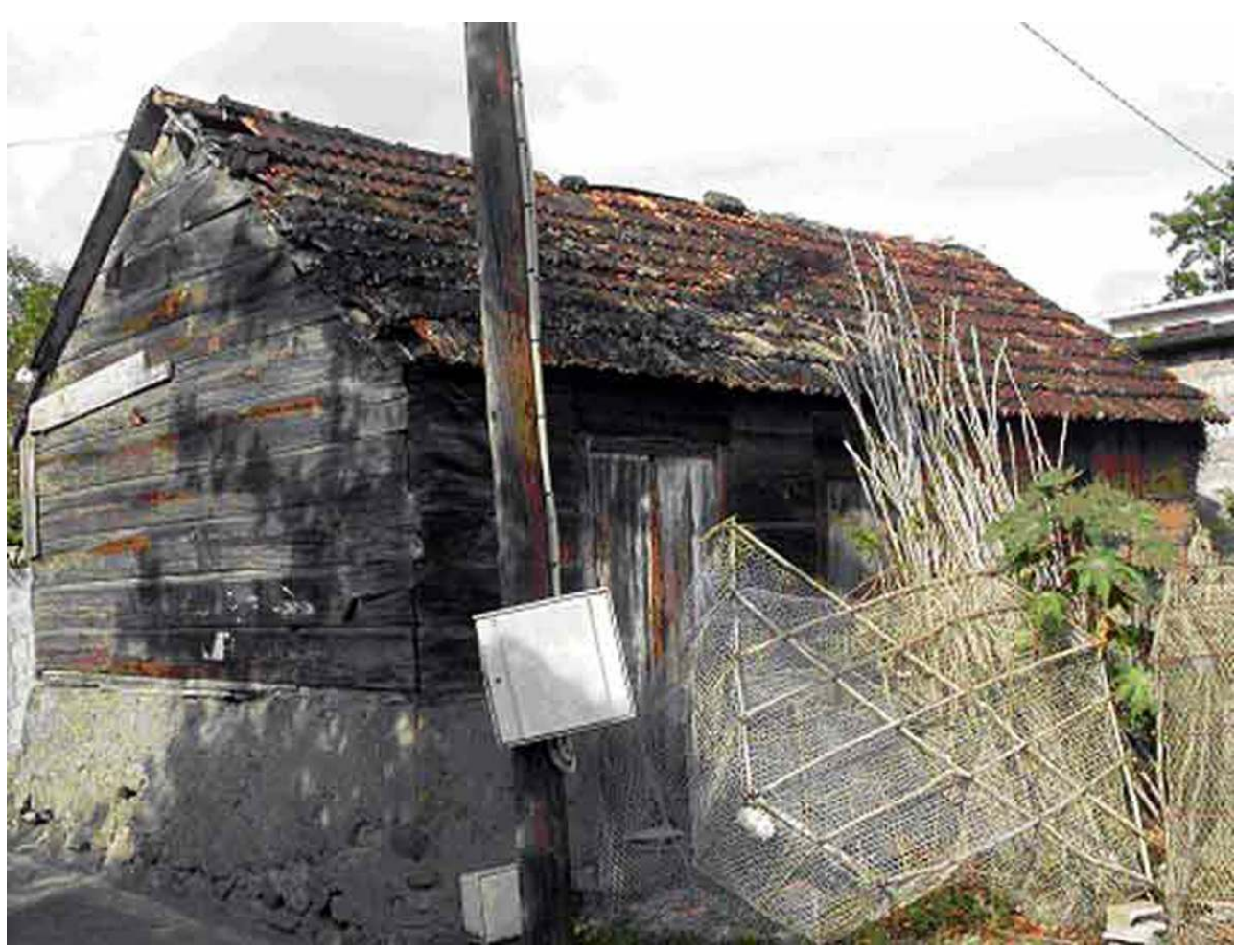

Case-Pilote, maison, rue de l'Autre Bord, premier quart du XX' siècle

Phot. Inv. O. Régis @ Inventaire général, ADAGP, 2003

La case est caractérisée par son plan et sa conception (fig. $\mathbf{n}^{\circ}$ 2). Delawarde en fait la description suivante : «La case accuse partout le même plan rectangulaire d'environ trois mètres sur cinq ou quatre mètres sur six et même huit de longueur. Elle est couverte d'un toit à deux pans retombant à hauteur d'homme, avec une forte inclinaison de quarantecinq degrés exigée par l'abondance des pluies et le chaume employé qui pourrit vite. Sa surface est partagée dans la longueur de deux pièces. Sur la première, s'ouvre la porte, c'est la salle à manger ou de réunion; la seconde, servant de chambre à coucher, ne communique pas avec l'extérieur. Deux ou trois baies carrées, placées comme les portes, contre les poteaux de la case, éclairent l'intérieur. Les principales ouvertures sont orientées vers le sud ou le couchant, surtout dans les endroits découverts que balaient les alizés ». Si l'agencement des baies reste libre, deux configurations prédominent: une fenêtre accostée d'une porte (généralement pour les cases en gaulettes) ou une porte flanquée de deux fenêtres (pour les cases en bois). Au-delà de cet aspect, la case s'intègre dans un ensemble. Elle s'inscrit en fait dans un espace aménagé pour répondre aux besoins et aux activités quotidiennes des habitants. Economiquement, la case est un outil de subsistance, d'indépendance. C'est un ensemble rigoureusement structuré. Aussi, « on doit moins parler de la case et ses espaces annexes que d'un espace habité dont la case ne serait qu'une partie $»^{10}$. Cet aménagement comprend: la case et un certain nombre d'espaces : la cuisine, située sous le vent afin de prévenir les incendies, un abri pour les éventuels animaux (porcs ou autres), le jardin créole (cultures vivrières), les toilettes 
rajoutées par la suite. La case peut alors se définir comme une « unité de production et de consommation organiquement liée à son environnement économique ».

\section{Les différents types de construction}

11 Construire une case, c'est s'adapter à son plus proche environnement. «(...) Parce qu'elle trouve ses matériaux dans le milieu même où elle s'élève, la case s'identifie au pays et varie d'aspect comme ce dernier ${ }^{11}$. En témoigne, la région du Nord où J.-B. Delawarde indique que : « (...) Les bambous poussant en abondance, on les utilise largement après les avoir fendus en lamelles (...) On en tresse des entrelacs dont l'étanchéité est assurée grâce à des «pailles cocos» qui sont les nervures des folioles des cocotiers, des "pailles cannes ", des " têtes roseaux » que l'on y introduit ». Ici, le bambou est alors utilisé tant pour fabriquer des palissades que des toitures.

12 Dans les régions où les champs de canne abondent: «(...) La case apparaît désormais comme un nid de paille. Les feuilles de cannes sont utilisées tant pour la toiture que pour les palissades, elles couvrent tout et pendent comme une chevelure $»^{12}$.

Figure 3

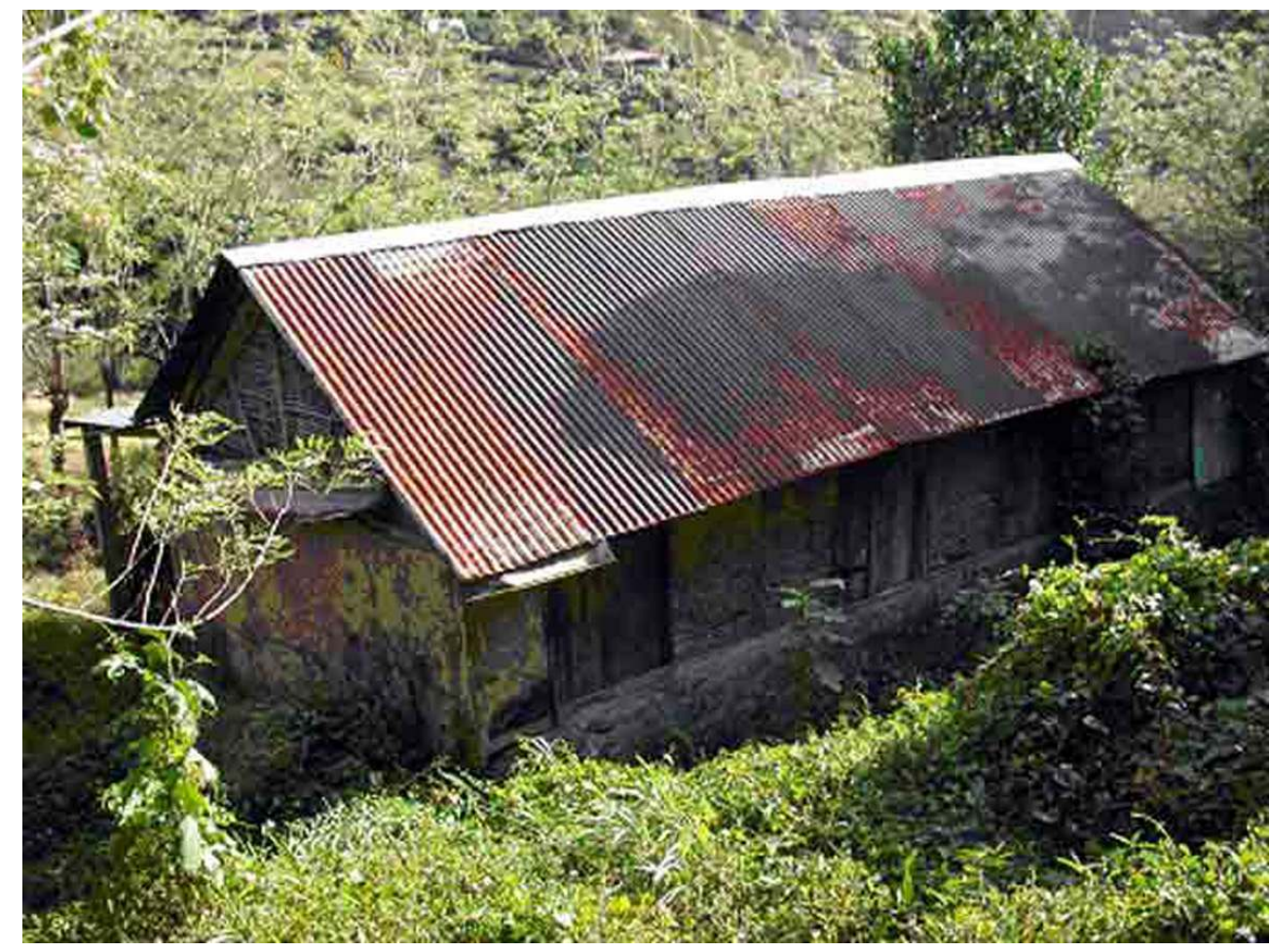

Morne-Vert, maison, route départementale 19, premier quart du XXe siècle

Phot. Inv. O. Régis (C) Inventaire général, ADAGP, 2003

13 Toutefois, le modèle de base est la case en " gaulettes » (fig. $\left.\mathbf{n}^{\circ} \mathbf{3}\right)$, recouverte de canne ou de vétiver. Il s'agit de branchages de ti-baumes tressés enduits d'un torchis. Les gaulettes sont recouvertes « d'une couche de terre grasse ou plutôt d'un épais mélange de bouse de vache, de cendre et de chaux éteinte, garantissant de la chaleur. Le piéton est presque la perfection dans ce genre ; il est fait de pieux fichés en terre et reliés par des rameaux et des lianes servant d'armature sur laquelle on coule et l'on plaque avec les mains, un pisé 
pétri, où de petites racines et des herbes sèches sont mêlées à la terre grasse pour mieux la lier. Ce travail se fait par coups de main ; le voisinage étant réuni, le tambour « bel air " donne le signal et l'on danse à la cadence de l'orchestre rural, les pieds et les jambes nus dans la pâte à pétrir. Les murs épais que l'on obtient ainsi conservent dans la case la fraîcheur matinale et, si l'on prend soin de les badigeonner avec une terre blanche délayée ou de peu de ciment, ils prennent un aspect décent. Si par ailleurs un avant-toit les protège contre les pluies, ils peuvent durer dix et vingt ans $»^{13}$.

Figure 4

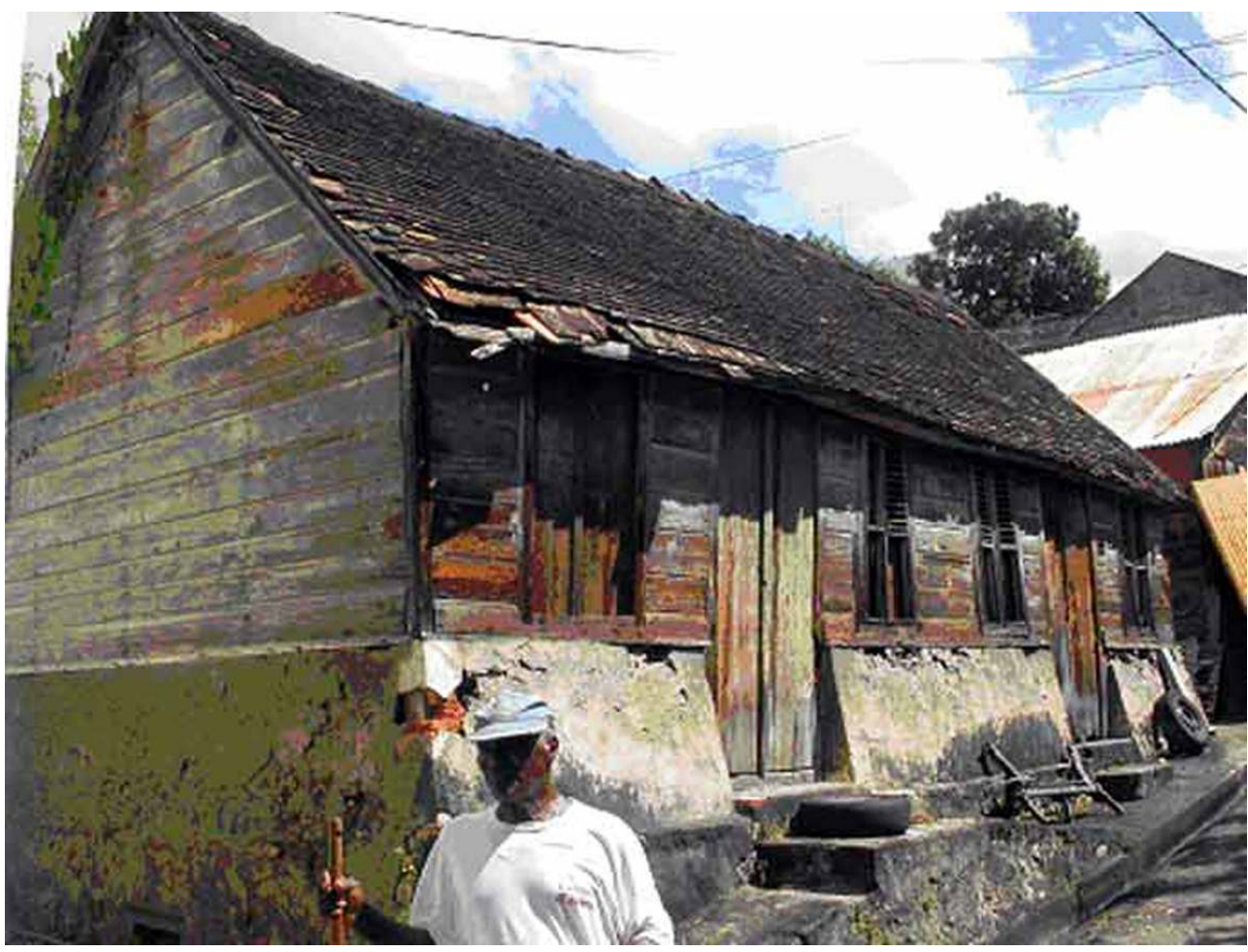

Carbet, maison, rue du Commandant Paraclet, premier quart du XXe siècle Phot. Inv. O. Régis (c) Inventaire général, ADAGP, 2003

On construit aussi la case avec du bois (fig. $\left.\mathbf{n}^{\circ} 4\right)$. A partir du second quart du XIX ${ }^{e}$ siècle, le modèle en "gaulettes " va disparaître au profit du bois. La généralisation du bois correspond à une hausse du pouvoir d'achat. Même s'il s'agit de bois pays, la construction d'une case en bois est d'un coût plus élevé, elle demande parfois le recours à des professionnels. Delawarde précise que «l'acajou, le balata, l'amer simarouba, l'incorruptible acouma ou acomas le roi des arbres à bâtir, l'angelin, les fougères arborescentes, le «bois lézard", le courbaril et plusieurs autres essences sont des matériaux de choix. Toutefois, l'arbre à pain, «l'abricotier pays ", le " poirier pays », le «laurier fine », le « laurier montagne », le « laurier rose », les durs lépinés, le «bois de rivière ", sont d'un bon usage et plusieurs de ces espèces ont l'agrément d'être parfumées et teintées comme les essences les plus rares. De nos jours, on achète et utilise beaucoup le « bois du Nord » importé d'Amérique, particulièrement de Floride ». Les élévations sont dans certains cas recouvertes d'essentes (écailles de bois) pour la protection contre l'humidité et la pluie. 
15 Autre caractéristique des cases martiniquaises, un solin de mur en maçonnerie sépare les élévations du sol. Héritage de la colonisation française, il permet de protéger ces dernières de l'humidité. On les réalise à l'aide de roches basaltiques récupérées dans les rivières auxquelles on associe un mortier, anciennement réalisé en mélangeant du sable ferreux à de la chaux et à des cendres de bagasses, auquel se substitua le ciment. Parfois, un soubassement en maçonnerie composé de roches juxtaposées puis liées peut servir à racheter la dénivellation du sol. La technique de la charpente marine est aussi une autre constante de l'architecture de la case. Il s'agit en fait d'une structure chevillée, assemblée à tenons et mortaises. Dans « la vie paysanne » Delawarde écrit : « Seule la charpente est réservée à l'ouvrier spécialisé.(...) C'est lui qui choisit les bois et dresse l'ossature de la case. Les poteaux sont passés au feu à leur base, à moins que leur bois ne contienne « un sirop ", une sève, qui le conserve dans le sol. Ils sont enfouis, non équarris ou à peine, à une profondeur de deux pieds et s'élèvent de cinq environ. Sommiers ou entraits, poinçons, arbalétriers, faîtage, chevrons, dessinent bientôt une carcasse légère, souvent imparfaite, retenue par de tenons et des mortaises taillées grossièrement à la hachette ou au coutelas ».

16 Cet habitat, ainsi décrit dans ses grandes lignes présente l'avantage de pouvoir être aisément transformé en fonction des souhaits et des besoins des habitants. Aussi, à partir du modèle traditionnel exposé ci-dessus, la case va évoluer avec l'aisance des paysans, sans toutefois perdre ses caractères essentiels.

\section{Aménagements et évolution}

De manière globale, la généralisation du bois a entraîné un certain nombre d'aménagements. L'ajout de parties supplémentaires au modèle de base fait apparaître de nouveaux types de cases. Souvent, l'édifice primaire est progressivement agrandi en fonction des ressources financières du moment. La case change alors de dimensions, qu'on lui adjoigne un espace de toilettes, ou bien une ou deux pièces supplémentaires. Un appentis peut être créé à l'avant de la maison. En le fermant, on obtient une galerie pouvant contenir une ou deux pièces. Parallèlement, l'espace extérieur peut aussi subir des modifications. On peut ainsi noter l'apparition d'espaces de confort ou de détente (galerie ouverte). 


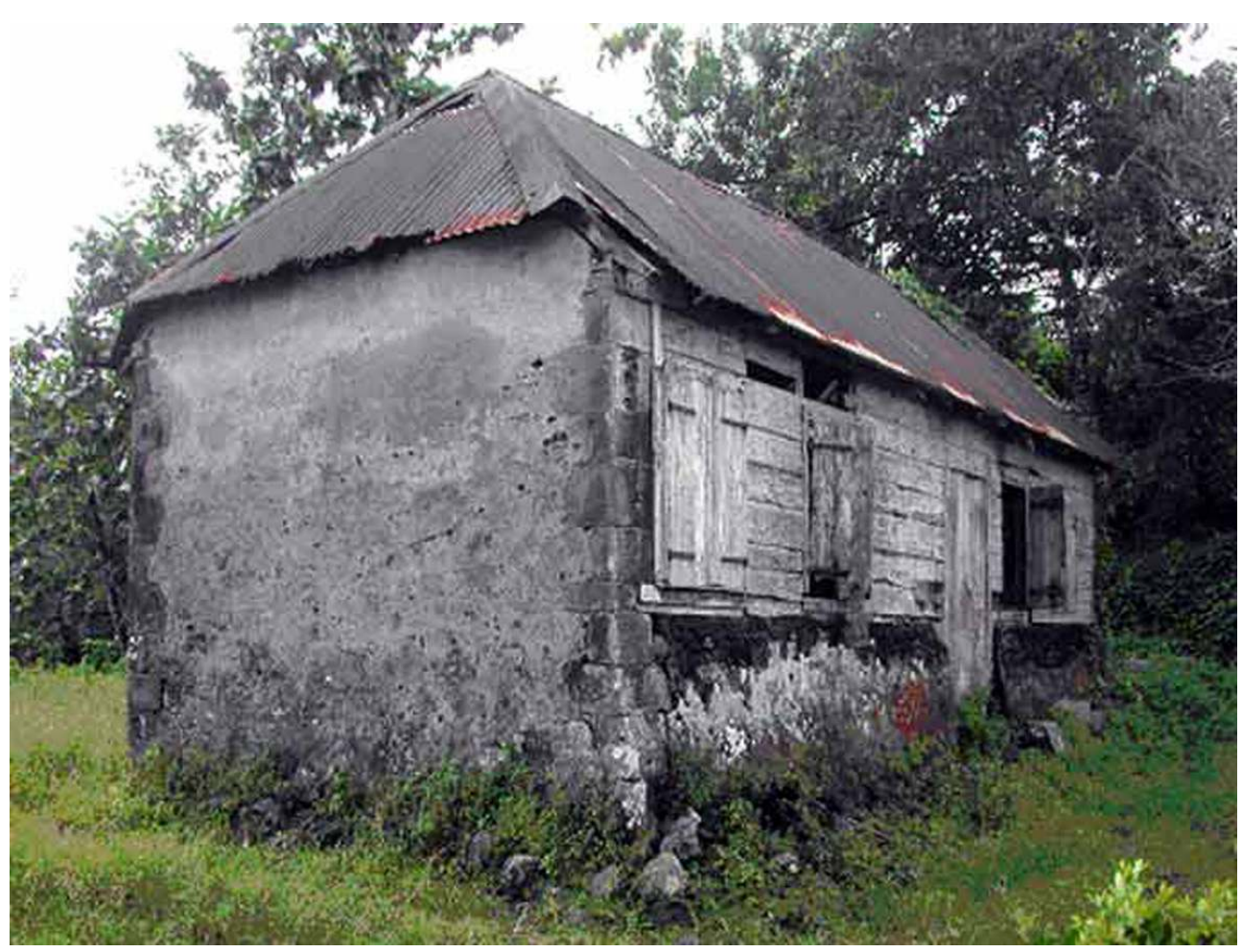

Ajoupa-Bouillon, maison, route nationale 3, premier quart du XXe siècle

Phot. Inv. O. Régis @ Inventaire général, ADAGP, 2003

En ce qui concerne les matériaux, on observe l'apparition du ciment dans la fabrication des solins de mur en maçonnerie ou d'enduit (fig. $\mathbf{n}^{\circ}$ 5), de la tôle ou plus rarement de la tuile mécanique en remplacement de la couverture en paille. A ce sujet, Delawarde affirme que « (...) La case évolue. Dans le passé déjà, l'emploi de la paille de canne avait été une sérieuse amélioration. Aujourd'hui, celle-là ne satisfait plus. La toiture de paille dure trop peu et présente des dangers d'incendie tandis que la tôle, qui rompt le charme du pittoresque, apparait comme une providence dans la vie pratique : le transport et la pose en sont faciles, la durée satisfaisante quand on est suffisamment écarté des côtes orientales d'où vient l'air salin. Et puis il y a une légitime ambition qui milite pour elle, le petit cultivateur est fier quand il construit une case de planches couvertes de tôles, en attendant mieux. A cette fin s'il le faut, il fera effort pour emprunter ». Depuis le second quart du XIX $\mathrm{X}^{\mathrm{e}}$ siècle, les toitures de paille n'existent déjà plus et à partir du milieu du siècle nous assistons à un phénomène général de « durcification ».

19 Comme nous l'avons souligné plus haut, dans l'imaginaire collectif martiniquais la case est un logement de pauvre. Aussi, dès qu'elle en a les moyens, une partie de la population éprouve-t-elle le besoin de s'émanciper par la «durcification». A partir de la seconde moitié du $\mathrm{XX}^{\mathrm{e}}$ siècle, l'augmentation du niveau de vie accentue la transformation du paysage rural : de la maisonnette on passe à la maison individuelle construite en béton armé. Ce changement introduit une mutation du mode d'habiter et plus généralement du paysage martiniquais. Les rapports entre villes et campagnes sont profondément transformés; force est de constater que depuis quelques années, on peut difficilement parler d'un habitat proprement rural, en ce sens qu'il n'y a pas de différence dans la manière de construire en ville et à la campagne. 


\section{NOTES}

1. Delawarde, Jean-Baptiste. La vie paysanne à la Martinique. Fort-de-France, 1937, p. 36.

2. Delawarde, Jean-Baptiste. La vie paysanne à la Martinique. Fort-de-France, 1937, p. 56.

3. Voir dans ce numéro l'article de Laurent-Christian Ursulet, « L'habitation Beauséjour ».

4. Begot, Danielle. Usines et habitations-sucreries, Trois siècles de patrimoine industriel martiniquais. Bureau du patrimoine du Conseil Régional de la Martinique, 1989, p. 27.

5. Petitjean-Roget, Jacques. Les premières habitations de la Martinique. Revue des Monuments historiques, $1981, \mathrm{n}^{\circ} 117$, p. 37-49.

6. Begot, Danielle. Usines et habitations-sucreries, Trois siècles de patrimoine industriel martiniquais. Bureau du patrimoine du Conseil Régional de la Martinique, 1989, p. 75.

7. Du Tertre, Jean-Baptiste. Histoire générale des Antilles habitées par les Français. Paris : E. Kolodziej, 1978, rééd. de 1667, p. 65.

8. Du Tertre, Jean-Baptiste. Histoire générale des Antilles habitées par les Français. Paris : E. Kolodziej, 1978, rééd. de 1667, p. 31.

9. Berthelot, Jack, GAUME, Martine. Kaz antiyè, jan moun ka rété. 1989, p. 43.

10. Berthelot, Jack, GAUME, Martine. Kaz antiyè, jan moun ka rété. 1989, p. 12.

11. Delawarde, Jean-Baptiste. La vie paysanne à la Martinique. Fort-de-France, 1937, p. 113.

12. Delawarde, Jean-Baptiste. La vie paysanne à la Martinique. Fort-de-France, 1937, p. 72.

13. Delawarde, Jean-Baptiste. La vie paysanne à la Martinique. Fort-de-France, 1937, p. 63.

\section{RÉSUMÉS}

Au cœur de l'habitat rural martiniquais se trouve la case. Elle s'inscrit dans un ensemble (case et dépendances) qui fait appel à une gestion de l'espace. Son architecture est intimement liée au mode d'habiter et par là même aux rapports qu'entretient l'individu avec la société. Si au cours de son évolution, la case a connu bon nombre d'aménagements, les caractères essentiels de cet habitat ont été préservés. Face à la transformation qu'a connu le paysage rural martiniquais depuis le milieu du $\mathrm{XX}^{\mathrm{e}}$ siècle, elle reste un héritage culturel caractéristique du langage architectural créole, patrimoine historique des anciennes sociétés coloniales qu'il convient de protéger.

In the heart of the rural settlement, there is the hut. It takes its place in a whole (hut and outbuildings) which has recourse to a space management. Its architecture is linked to the mode of life and the individual's relations with the society. If in its evolution the hut undergone many adjustments, its essential characteristics have been preserved. Facing the transformations of the rural settlement since the middle of the twentieth century, it stays a cultural legacy characteristic of architectural creole language, historical patrimony of the ex-colonial societies which should be protected. 
INDEX

Mots-clés : Caraïbe, case, cases-nègres, colonial, créole, gaulettes, habitation, martiniquais, Martinique, Mouinas, Plantation, sucrerie

\section{AUTEUR}

\section{CHRISTOPHE DENISE}

Chercheur, Service régional de l'Inventaire, Direction régionale des affaires culturelles de Martinique. invite.martinique@culture.gouv.fr 